\title{
Uso de tertulias dialógicas. Resultados en los exámenes de ciencia agraria
}

\author{
Isabel López-Cortés ${ }^{a}$, Domingo M. Salazara ${ }^{a}$, Borja Velázquez Martíb, Javier Estornell \\ Cremades ${ }^{c}$, Juan Martínez-Toméd \\ ${ }^{a}$ Dpto Producción Vegetal COMAV Universitat Politècnica de València. Valencia. España \\ islocor@upv.es, bDpto. Ingeniería Rural y Agoralimentaria. Universitat Politècnica de València. \\ Valencia. España, ${ }^{\mathrm{c} D p t o . ~ I n g e n i e r i ́ a ~ c a r t o g r a ́ f i c a, ~ g e o d e s i a ~ y ~ f o t o g r a m e t r i ́ a . ~ U n i v e r s i t a t ~ P o l i t e ̀ c n i c a ~ d e ~}$ \\ València. Valencia. España, ${ }^{\mathrm{d}}$ Dpto. Producción Vegetal y Microbiología. Universidad Miguel \\ Hernández. Orihuela. España.
}

\begin{abstract}
Resumen
Actualmente en las asignaturas de origen agrario es totalmente necesario utilizar técnicas prácticas. Se ha comprobado a lo largo de los años que son necesarios enfoques que permitan disponer de más horas prácticas para aplicar, con más rapidez y fiabilidad, cualquier técnica agraria que estemos estudiando en las aulas. Como también queda reflejado en los procesos de renovación docente de las enseñanzas universitarias, estas técnicas son necesarias para producir el cambio educativo.

Con las tertulias dialógicas se trata de continuar la construcción del conocimiento a partir del diálogo que inició el autor al escribir su obra, primero de una forma individual, para pasar después a enriquecerlo aún más a través de un diálogo colectivo, diálogo que debe facilitar un conocimiento no adquirido. A la ve zque con un sistema participativo y nuevas dinámicas de grupo, conseguiremos que el alumno adquiera conocimientos y los afiance.
\end{abstract}

Las tertulias fueron desarrolladas en asignaturas diferentes y aunque las tenían un claro perfil agrario y se encontraban incluidas en un mismo plan de estudios. Permitió establecer resultados diferentes dado que la temática de las asignaturas lo permitía.

Las asignaturas elegidas son de diferentes cultivos, leñosos y herbáceos, así como de su manejo Todas ellas en la misma titulación y en diferentes centros. Realizando por lo tanto en distintos enfoques de alumnado $y$ contextos muy diversos, tanto formal como no formal. 


\section{Los alumnos obtuvieron mejores resultados cuando la temática era tratada mediante tertulia dialógica dado que conseguía emular las condiciones prácticas con la búsqueda de información a través de la lectura.}

Palabras clave: Tertulia, Diálogo, Unidades temáticas, Cultivos agrarios.

\section{Introducción}

Los diferentes cambios en la Universidad en los últimos años han generado que seamos conscientes de que el nivel de competencias que las personas acreditan es más importante que el título que estos alumnos obtengan (Rekalde et al, 2004). Por ello, es interesante buscar formas de aprendizaje que no se basen en técnicas memorísticas y que permitan construir el conocimiento a partir de conocimientos ajenos, con los que puedas estar de acuerdo o no.

Es necesario establecer una reflexión dentro de la actual situación educativa y social de las universidades. Por es es interesante analizar el sentido y la función que las universidades cumplen en el contexto socioeducativo actual. Al mismo tiempo es necesario aplicar y contrastar la utilización de métodos alternativos o distintos a los que acutalmente están siendo utilizados en las aulas universitarias (Arandia et al, 2008).

La enseñanza universitaria tiene que ir más allá del conocimiento de la temática. La labor de un professor es formativa, pero no puede solo plantear contenidos sino también debe establecer cómo trabajar con estos contenidos en el aula, de esta forma se consiguen clases mas dinámicas y cercanas al alumno (Martínez et al, 2011).

La tertulia dialógica es un proceso establecido entre leer y comprender un texto, es un proceso en el que los alumnos profundizan mediante sus interpretaciones en las temáticas concretas de los temas agrarios, que forman parte de las unidades temáticas de nuestro plan de estudio.

Está claramente comprobado que cada alumno desarrolla un aprendizaje diferente en cada lectura y en cada entorno dialéctico que se plantee. Bajo esta premisa, se plantean las interacciones profesor-alumno y alumno-artículo para lograr crear de esta forma interacciones entre el mundo agrario y el mundo académico. Es importante correlacionar de manera directa ambos aspectos del sector agrario.

Por todos estos motivos, nos planteamos en su momento incorporar en nuestra docencia no solo nuevas metodologías sino ante todo nuevas fuentes de información. Introducidas estas fuentes de información mediante el uso de las tertulias dialógicas. Decidimos comparar nuestro sistema previo con el sistema de tertulia dialógica. Y lógicamente la forma más 
rápida de poder constrastar esa situación es a través de los sistemas de evaluación. Por ello establecimos una comparación entre unidades temáticas en la evaluación, de modo que las unidades las desarrollamos con tertulias dialógicas y sin este apoyo.

Lo importante era lograr que el alumno interaccione con la realidad agraria a través de la lectura preparada para cada unidad temática. Es igualmente importante la herramienta usada como lo que hacemos con ella (Alonso et al, 2006) esto se consigue comprobar con todas las aportaciones que los estudiantes van creando alrededor de las unidades temáticas.

Otro punto importante, es acercar las investigaciones agrarias al alumno por lo que dentro de un marco comunicativo necesitamos buscar nuevas metodologías, de ahí nuestro interés en las tertulias dialógicas en la enseñanza universitaria (Aguilar, 2004; Boscolo, 2005).

A lo largo del curso se plantearon dos tipos de unidades temáticas, unas de ellas siguieron el esquema habitual y el desarrollo tradicional de la clase. Mientras otras unidades temáticas se plantearon en todo su desarrollo como unidades con tertulias dialógicas.

En este segundo caso se facilitó al alumno entre tres y cinco lecturas sobre la temática de la unidad temática. Se decidió facilitar más de un artículo intentando de esa forma motivar al alumno a terminar las lecturas, y que el alumno eligiera cuál de todas ellas le resultaba más interesante.

\section{Material y Métodos}

Al comienzo del curso repartimos las unidades temáticas entre unidades con metodología de tertulia y otras con dinámica docente tradicional. El tamaño pequeño de nuestros grupos de clase puede verse como un aspecto inadecuado, sin embargo, nos permitió comprobar la idoneidad de las tertulias dialógicas en docencia agraria.

La distribución de la unidades temáticas fue totalmente aleatoria, nos interesaba describir si realmente las tertulias resultan útiles o sin embargo, teniamos cierta influencia según el gusto personal de los alumnos por una unidad temática concreta. Por este motivo, la decision de qué unidades se incorporaron en cada formato en nuestras clases, fue totalmente indiscriminada, para que no puediera influir temas socialmente más activos o dinámicos por existir sobre ello más investigación, o que los temas se vieran influenciados por la política o dinámica social del momento.

Se plantearon a los alumnos un grupo de artículos, entre 3 y 4 , para que sea el alumnado quien decida qué publicación, web o artículo le resulta más interesante para empeza a conocer un tema, en principio novedoso hasta ese momento. 
Como vemos en la figura 1 los alumnos tienen claras preferencias en cuanto al origen de la información.

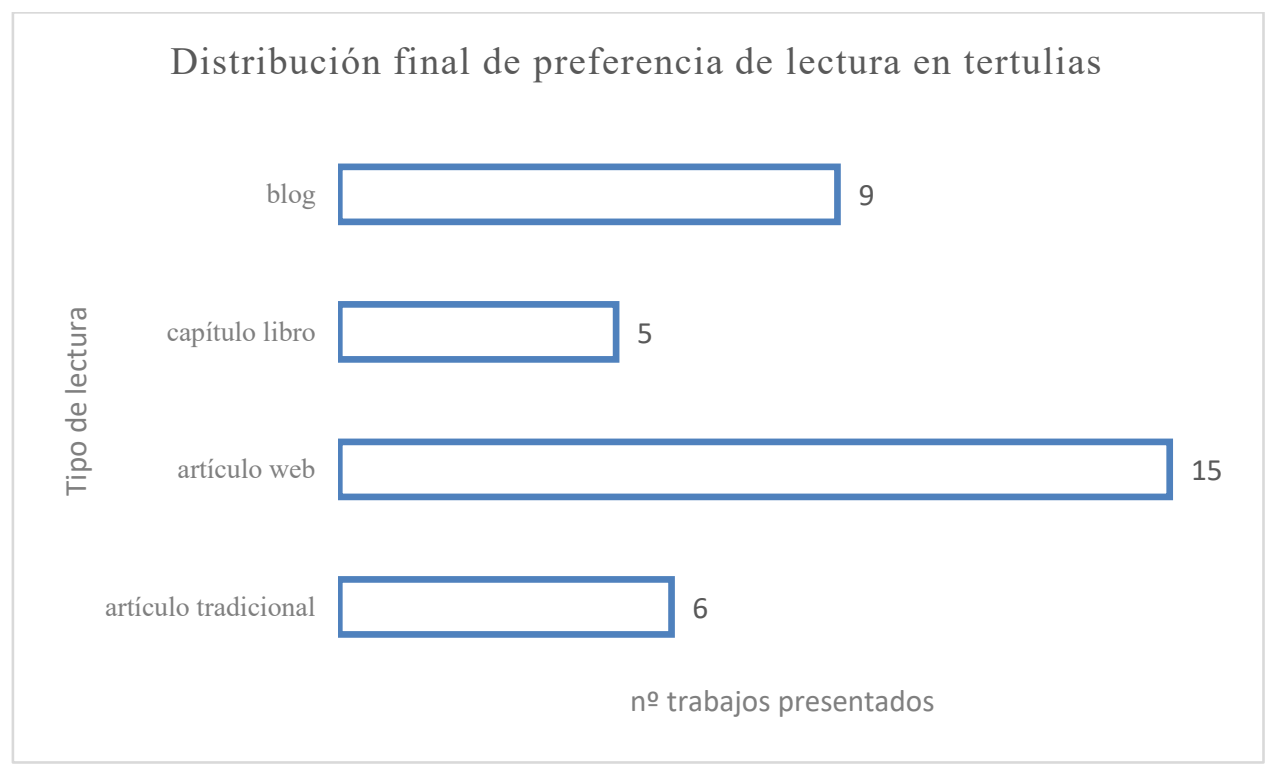

Fig. 1 Distribución de origen final de los articulos utilizados en las tertulias

Este trabajo ha sido realizado en dos momentos diferentes del curso. Esta decision se tomó por dos motivos distintos. En primer lugar, repartimos las unidades temáticas en función de la adaptación de las mismas al ciclo fenológico de las plantas en el cultivo que se impartía en aula. Este aspecto es importante en asignaturas agrarias dado que ello permite estudiar en la asignatura todas las fases de desarrollo del cultivo.

En segundo lugar, el interés se centraba en poder retoralimentar la prueba. Este segundo motivo generó la posibilidad de dejar elegir al alumnado sus preferencias, en cuanto al formato en el que prefiere realizar las lecturas, para en la segunda fecha disponer, en el formato de su elección de suficientes artículos, para que esto no fuera un punto negativo a la hora de tomar una decisión de participación.

Hemos comprobado que influye el soporte en el que se presenta la lectura, se puede constatar claramente una relación estrecha entre el alumno y la lectura presentada. Por este motivo se plantearon las lecturas mediante la plataforma de la Universidad. De esta forma, resulta más fácil conseguir que el alumno accede a la lectura y en algunos casos les facilita una confianza que permite asegurarse el seguimiento por su parte. 
Finalmente, con toda esa información hemos comparado la evaluación de esas unidades temáticas y su repersución en la nota final (figura 2). De esta forma hemos podido establecer que hay diferencias claras entre el uso de las tertulias para el desarrollo de las Unidades Temáticas frente a la clase tradicional, en asignaturas de carácter agrario como las que nos ocupan.

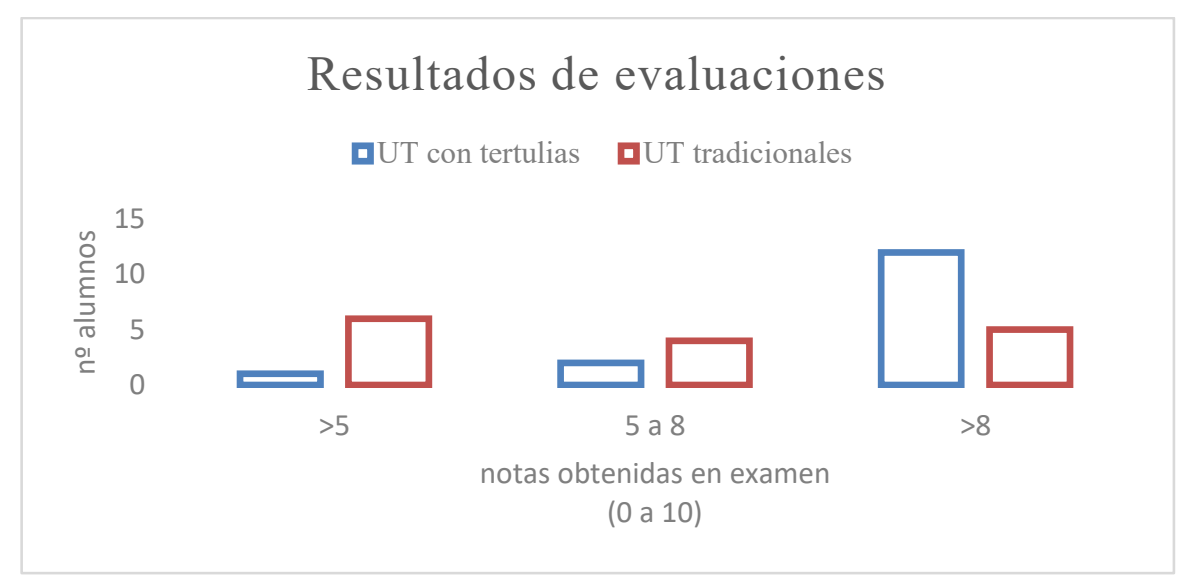

Fig. 2 Distribución de notas según Unidad Temática (valoraciones sobre 10)

Sería interesante desarrollar estas tertulias dialógicas en otro tipo de asignaturas que permitan interrelacionar más temáticas divugadoras y que permitan interrelacionar temáticas practicas con los enfoques puramente teóricos.

\section{Conclusiones}

Tras el desarrollo del curso hemos conseguido obtener una lectura igualitaria lo cual es una de las premisas de las tertulias dialógicas.

Se ha conseguido a lo largo del curso, que los alumnos opinen abiertamente sobre la temática de la unidad, a la vez que sus reflexiones son un punto de origen para desarrollar más temas de discusión y por lo tanto de investigación.

Los resultados finales obtenidos en la evaluación de la asignatura son claramente superiores en el caso de las Unidades Temáticas con tertulia dialógica que las Unidades Temáticas tradicionales, con docencia presencial en aula y trabajo asincrónico. 
Los alumnos prefieren como formato de trabajo, aquellos que tienen origen en web y los blogs frente al artículo en pdf o papel.

\section{Referencias}

Aguilar, C. (2004). La tertulia literaria dialógica del CREA o cómo aprender a saltar los topes de la desigualdad social a través de la literatura. Actas del VII Congreso Internacional de la Sociendad Española de Didáctica de la Lengua ly la Literatura. 1(92). 617-625.

Arandia M., Alonso M., Martínez I. (2010). La metodología dialógica en las aulas universitarias. (2010). Revista de educación 352. 309-329.

Alonso, M.J., Del Castillo, L., Arandia, M.T., Martínez, I., Rekalde, I., \& Zarandona, E. (2012). El uso y experimentación de herrmientas comunicativas en un equipo Universitario de investigaciónacción. Revista Iberoamericana de Educación. 60 (4). 1-11.

Gómez, J., Flecha, R., De La Torre, A. y Sánchez, M. (2006). Metodología comunicativa crítica. Barcelona: El Roure.

Martínez, I., Arandia, M. T., Alonso, M. J., Del Castillo, L., Zarandona, E. \& Rekalde, I. (2011). Trabajar con metodologías participativas en la formación universitaria, todo un desafío. Investigación en la escuela, 75, 101-113.

Rekalde I., Alonso I., Arandia, M., Martínez, I., \& Zarandona, E. (2014). Las tertulias literarias dialógicas en los procesos de enseñanza universiarios: Reflexiones desde la práctica docente. Revista de formación e innovación educativa universitaria. 7 (3). 155-172.

Rekalde I., Alonso J., Arandia M., Martínez I., Zarandona E. (2014). Revista de formación e innovación educativa universitaria. 7 (3). 155-172.

Rodríguez M. (2014). El aprendizaje-servicio como estrategia metodológica en la Universidad. Revista complutense de educación. 25 (1). 95-113.

Teva, I., \& Buela-Casal, G. (2011). El proceso de enseñanza-aprendizaje y la actividad investigadora en el marco del Espacio Europeo de Educación Superior. Revista Bordón. 63 (2). 9-14.

Teale, W.y Sulzby, E. (1986). Emergent Literacy. Writing and Reading. Norwood, NY: Abblex.

Valero, L. F. y Brunet, I. (1999). Algunas consideraciones sobre la Universidad del siglo XXI. Revista electrónica Interuniversitaria de Formación del Profesorado, 2(1), 463-469. http://www.uva.es.

Valls, R., Soler, M., \& Flecha, R. (2008). Lectura dialógica: Interacción es que mejoran y aceleran la lectura. Revista Iberoamericana de Educación. 46. 71-87.

Wells, G. (1981). Learning Through Interaction: The Study of Language Development. Language at Home and School, vol. 1. Cambridge University Press.

Wells, G. (2001). Indagación dialógica. Hacia una teoría y una práctica socioculturales de la educación. Barcelona: Paidós. 